\title{
Agrice et les tensioactifs
}

Agrice and surfactants

Oléagineux, Corps Gras, Lipides. Volume 8, Numéro 2, 160, Mars - Avril 2001, Dossier : Tensioactifs : savons et détergents

Auteur(s) : Magali ROCHER, Ademe, Direction de l'agriculture et des bioénergies, 2, square La Fayette, BP 406, 49004 Angers Cedex 01.

Résumé : Agrice, Agriculture pour la chimie et l'énergie, est un groupement d'intérêt scientifique qui a pour objet l'animation, le financement, le suivi et l'évaluation de programmes de recherche et développement portant sur les nouvelles valorisations des produits et coproduits d'origine agricole dans les domaines de l'énergie, de la chimie et des matériaux. Aujourd'hui les programmes de recherche et développement sur les tensioactifs sont renforcés, plus sélectifs et plus directement tournés vers l'industrie et les marchés.

Mots-clés : agro-ressources, recherche et développement, tensioactifs

Summary : The scientific interest group AGRICE - Agriculture for Chemicals and Energy - focuses on new uses and enhanced value for agricultural products and byproducts as energy, chemical (surfactants) and materials feedstocks. AGRICE is committed to coordinating, funding, monitoring and evaluating research and development programmes that further these goals. Today surfactant's research-development programmes have been strengthened and fine-tuned, with a more direct focus on industry and markets.

Keywords : non food, research and development, surfactant.

\section{ARTICLE}

Abstract: The scientific interest group AGRICE - Agriculture for Chemicals and Energy - focuses on new uses and enhanced value for agricultural products and byproducts as energy, chemical (surfactants) and materials feedstocks. AGRICE is committed to coordinating, funding, monitoring and evaluating research and development programmes that further these goals.

Today surfactant's research-development programmes have been strengthened and fine-tuned, with a more direct focus on industry and markets.

Key words: non food, research and development, surfactant. 
Résumé : Agrice, Agriculture pour la chimie et l'énergie, est un groupement d'intérêt scientifique qui a pour objet l'animation, le financement, le suivi et l'évaluation de programmes de recherche et développement portant sur les nouvelles valorisations des produits et coproduits d'origine agricole dans les domaines de l'énergie, de la chimie et des matériaux.

Aujourd'hui les programmes de recherche et développement sur les tensioactifs sont renforcés, plus sélectifs et plus directement tournés vers l'industrie et les marchés.

Mots clés : agro-ressources, recherche et développement, tensioactifs.

Depuis 1994, le groupement d'intérêt scientifique Agrice (Agriculture pour la chimie et l'énergie) coordonne, finance et évalue des programmes de recherche portant sur les nouvelles valorisations des agro-ressources. Agrice réunit à la fois des organismes de recherche publics et privés. Parmi les thématiques développées, les tensioactifs ont fait l'objet de 30 projets pilotés par l'Ademe. L'aide totale apportée à ces projets en sept ans représente 2,7 millions d'euros pour un volume de recherche généré de 8,9 millions d'euros.

En raison de leur structure, les tensioactifs d'origine végétale permettent la valorisation de diverses matières premières extraites, entre autres, de betterave, de blé, de colza, de maïs, et de tournesol. Les spécifications des tensioactifs développés dans les projets Agrice doivent, en termes de fonctionnalité, être au moins équivalentes à celles des dérivés pétrochimiques et supérieures d'un point de vue environnemental. Outre leur participation à la lutte contre l'épuisement des ressources, l'effet de serre et la pollution, ils sont caractérisés par un impact favorable sur l'environnement (nonécotoxicité, biodégradabilité) ainsi que par une innocuité pour la santé humaine.

Les deux tiers des projets soutenus par Agrice portent sur la synthèse de nouveaux produits aux propriétés tensioactives, plurifonctionnels et respectueux de l'environnement. La recherche et le développement de propriétés additionnelles de ces molécules respectueuses de l'environnement permettent d'espérer une compétitivité par rapport aux dérivés pétrochimiques ainsi qu'une simplification des formulations. Les valorisations envisagées pour ces nouvelles molécules concernent majoritairement les secteurs de l'hygiène, de la cosmétique et de la pharmaceutique. En effet, aujourd'hui, les agro-tensioactifs conduisent principalement à des produits de haute valeur ajoutée. II existe des productions industrielles encourageantes d'alternatives végétales dans ces secteurs. Certains programmes Agrice concernant les nouveaux agrotensioactifs ont abouti à des dépôts de brevet, et des développements commerciaux sont en cours.

D'autres travaux de recherche ont permis, dans un deuxième temps, le passage de projets du stade laboratoire au niveau pilote. Ainsi, des secteurs permettant de produire de plus grands volumes à de moins hautes valeurs ajoutées ont été explorés. Aujourd'hui, des agrotensioactifs identifiés dans le cadre d'Agrice ont débouché sur les marchés des composants de formulations phytosanitaires et des fluides de forage pétroliers. La nécessaire collaboration aux programmes d'un utilisateur positionné sur le marché d'application a clairement été démontrée par les résultats obtenus jusqu'ici. Cette logique de collaboration s'avère être une condition quasi indispensable au succès.

Le troisième volet des actions soutenues par Agrice concerne le développement, la simplification ou l'optimisation de procédés de synthèse chimique ou microbiologique d'agro- tensioactifs. La recherche de compétitivité économique et technique vis-à-vis des procédés pétrochimiques est alors 
associée au respect de l'environnement. Ces études ont permis d'identifier et de limiter les réactions secondaires parasites aux procédés industriels de fabrication mais également de réduire les étapes de purifications et le volume de déchets générés.

Cette thématique est suivie au sein d'Agrice par un groupe de travail associant producteurs, applicateurs et utilisateurs de tensioactifs mais également centres de recherche. Dans ce cadre, une réflexion est menée pour établir les axes de recherche prioritaires et déterminer les solutions compétitives et respectueuses de l'environnement pour les années à venir. 